\title{
NEUTRON DEPTH PROFILE CALCULATIONS USING ARTIFICIAL NEURAL NETWORKS
}

\author{
K. Hossny' ${ }^{1}$, S. Magdi' ${ }^{2}$ F. Nasr'², Y. Yasser ${ }^{2}$, and A. Magdy ${ }^{3}$ \\ [1] Basic Sciences Department, Faculty of Engineering, The British University in Egypt (BUE), \\ Sherouk City, Cairo, Egypt. \\ [2] Nuclear and Radiation Engineering Department, Faculty of Engineering, Alexandria \\ University, Alexandria, Egypt. \\ [3] Faculty of Engineering, Alexandria University, Alexandria, Egypt.
}

Karim.Hossny@,BUE.edu.eg, salmamagdi2004@yahoo.com, eng-fadel.nasr1520@alexu.edu.eg, eng-youssef.yasser1621@,alexu.edu.eg, abdomagdy816@gmail.com

\begin{abstract}
Abstract: Neutron depth profiling (NDP) is a non-destructive technique used for identifying the concentration of impurity isotopes below the sample surface. NDP is carried out by detection of the emitted charged particles resulting from bombarding the sample with neutrons. NDP specifies the isotopic concentration versus the sample depth for a few micrometers below the surface. The sample is bombarded inside a research reactor using a thermal neutron beam. Charged particles like alpha particles or protons are produced from the neutron induced reactions in the sample. Each neutron isotopic interaction produces a certain $\mathrm{Q}$, indicating a specific kinetic energy for the emitted charged particle. As the charged particle travels through the sample to eject the surface, it loses energy to atoms (electrons) on its path. The charged particle energy loss holds information regarding the number of atoms by which the emitted particle passed, thus indicating its original depth. The purpose of this work is to check the capability of Artificial Neural Networks (ANNs) in predicting the boron concentration profile across a boro-silicate sample of thickness 3.5 $\mu \mathrm{m}$ divided into 10 layers. Each layer included different boron concentration than the other. Also, the boron concentration had the values $\{0.1,0.2,0.3,0.4,0.5,0.6,0.7,0.8,0.9,1\}$. Training, validation, and test data were generated synthetically using MCNP6 in which the boron concentrations varied in the layer number from one sample to another. MCNP6 model consisted of a silicon barrier detector, boro-silicate sample, chamber body and an idealized thermal neutron source. The detector, sample, and the source were located in a voided chamber. The samples were irradiated with a $0.025 \mathrm{eV}$ monoenergetic thermal neutron beam from a monodirectional disk source. To cover the whole area of the samples, the thermal neutron beam had a radius of $3 \mathrm{~cm}$. The silicon detector active volume was modelled as a $100 \mu \mathrm{m}$ thick and $3 \mathrm{~cm}$ radius facing the sample directly. The sample, beam, and the detector were placed on the same axis. Ten ANN regression models were developed, one for each layer boron concentration prediction where the input for each model was the alpha spectrum read by the detector, while the output was the boron concentration for each layer. Results showed regression values higher than 0.94 for all of the developed models. ANNs proved its capability of predicting the boron profile form the alpha spectrum read by the detector regarding neutron depth profiling in a boro-silicate samples.
\end{abstract}

KEYWORDS: Artificial Neural Networks (ANNs), Neutron Depth Profiling, MCNP6. 


\section{INTRODUCTION}

Neutron depth profiling (NDP) is a near-surface analytical technique used to determine the concentration profiles of an impurity isotope as a function of depth (concentration versus depth). It was first reported by Ziegler et al. [1] in 1972 as a near-surface technique which has come to be known as neutron depth profiling, and was later improved by Biersack et al. [2] to most of its existing capabilities. It is a non-destructive technique based on measuring the energy loss of a charged particle emitted from the tested sample. The specimen is bombarded using thermal or cold neutron beam, the interaction produces charged particles like protons and alpha particles $\left(\mathrm{H}^{+}, \mathrm{He}\right)$. Each nuclear reaction produces a certain Q-value, inducing a specific kinetic energy for the emitted charged particle. As the charged particle travels through the sample, it interacts with the electrons in its path and loses energy. The depth at which the charged particle originated can be deduced by the energy loss of the particle using the stopping power of the material. NDP is carried out by detecting these charged particles and measuring their residual energies. Analyzing the energy spectrum of the emitted charged particles gives information regarding the number of atoms by which the particle passed while exiting the substrate; thus indicating its original depth. [3]

The technique can be applied for certain light elements like He, Li, B, N, Cl etc. It can be used in polymer samples, optoelectronic materials, semiconductors, metal alloys, and other solids. It only can be applied below the surface up to a few tens of micrometers with a resolution up to a few tens of nanometers. [4]

\subsection{Neutron Depth Profiling Calculations}

NDP is carried out by producing a thermal neutron beam of cross-sectional area $A_{n}$ in a vacuum chamber with a uniform thermal flux $\phi_{t}$. The neutron beam illuminates the plane surface of the tested sample making an angle $\theta_{n}$ with the surface normal. The detector is assumed to have a plane surface area of $A_{d}$. Ions have a probability of reaching $A_{d}$ given by $P_{d}=\frac{\Delta \Omega_{d}}{4 \pi}$, where $\Delta \Omega_{d}$ is the solid angle subtended by the detector surface.

Conventionally, concentration versus depth is computed using NDP by assuming: 1) The thermal neutron flux $\phi_{t}$ experiences negligible attenuation when passing through the sample, 2) Produced ions are scattered isotropically, 3) All ions originating from the same depth and reaching the detector have the same energy, 4) The detector has perfect energy resolution, and 5) No energy broadening present in the measured spectrum.

The production rate of ions depends on the concentration of the isotope interacting with neutrons. Thus, the energy distribution $N(E)$ of ions escaping the surface of the sample can be used to determine the concentration profile $C(x)$ of the interacting isotope, where $\mathrm{x}$ is the isotope depth in the sample.

Depending on previously mentioned assumptions, the relationship between concentration profile and both energy distribution and stopping power is given by:

$$
C(x)=\frac{1}{\kappa} N(E(s)) S(E(s))
$$

Where

$$
\kappa=f_{\text {ion }} \bar{\sigma}_{\text {ion }} \phi_{t} \frac{A_{n}}{\cos \theta_{n}} \frac{\epsilon A_{d} \cos \theta_{s}}{4 \pi r_{d}^{2}}
$$

Given that:

1) $\bar{\sigma}_{i o n}$ is the microscopic thermal-averaged cross section for ion production.

2) $f_{\text {ion }}$ is the fractional yield for the ion of interest per ion production reaction.

3) $\mathrm{E}(\mathrm{s})$ is the residual energy of ion after moving a distance ' $\mathrm{s}$ ' in the tested material. 
4) $\mathrm{S}(\mathrm{E})$ is the ion stopping power measured in $\mathrm{MeV} / \mu \mathrm{m}$.

5) $\epsilon$ is the detector efficiency per incident ion.

6) $r_{d}$ is the sample to detector distance.

7) $\theta_{s}$ is the angle subtended between the sample normal and $r_{d}$ direction. [5]

\subsection{Artificial Neural Networks}

Machine learning is an application of artificial intelligence that provides systems with the ability to learn without explicated programming. Machine learning techniques are implemented using large sets of data, where the algorithm aims to achieve an accurate model connecting inputs and outputs. There are two methods of machine learning: 1) Supervised learning; where the algorithm learns to predict the output from the input, 2) Unsupervised learning; where the algorithm learns the structure of the system from the input data. [6]

Artificial Neural Network (ANN) is a type of supervised machine learning that tries to simulate the way the information flows in a human brain. ANN structure consists of an input vector $(\mathrm{X})$, weights $(\mathrm{W})$, biases (B), hidden layers (H), and output $(\mathrm{Y})$. The inputs are fed to an input neuron layer, followed by hidden layer (or layers) of neurons interconnected to generate the final output layer. Usually a bias neuron is added in each layer to allow flexibility of the output despite input restrictions, and different neurons are assigned different weights. Weights and biases are initialized randomly, weights are multiplied by the corresponding input value, then added to the biases, this is called the activation function. Neurons in the hidden layer make decisions to reach an optimal value of the output. This is done through three sets of data: 1) Training set, 2) Validation set, and 3) Testing set. The training set is given to the neural network where the inputs and outputs are known. The neurons make decisions and predict an output. The loss function is used to calculate the error between the predicted and actual output and is used to update the values of weights and biases using backpropagation. Backpropagation is done by performing a backward pass to find the optimal neuron weights which minimize the loss function and predict the best result. In this way, the ANN learns the features and patterns between these data and predicts an accurate output value. After training the neural network, a validation set is used to test the performance of the ANN. This is crucial to make sure the developed ANN does not overfit. Overfitting refers to poor performance caused by training the noise data as concepts in the model. Therefore, using these concepts to generalize a system will predict a highly defective output for new data. This can be detected in the validation set as the loss function increases. The testing set is used as a final step to compare and measure the performance of the network. [7]

\subsection{Literature Review}

V. Havranek et al. [8] used NDP analysis to demonstrate depth profiling of boron and lithium. They found the depth resolution was dependent on the analyzed depth and emission angle. Kenan Unlu et al. [9] worked in the University of Texas (UT) TRIGA Mark-II research reactor to determine the boron profiles of borophospho-silicate glass (BPSG), the radiation damage in stainless steel, and to measure the boron-10 implant concentration of semiconductor material. Sacit M. Cetiner et al. [10] successfully obtained concentration verses depth profiles of boron by using time-of-flight neutron depth profiling (TOF-NDP). It was found that the TOF method increases the energy resolution, in addition to giving more data points than those obtained by conventional methods, thus showing more features.

R. Bencardino et al. [11] used NDP to characterize LiF enriched to $95.5 \% \mathrm{Li}^{6}$ deposited in aluminum foils. NDP results agreed with the deposited layers; thus they were benchmarked with the standards of the Institute for Reference Materials and Measurements (IRMM). Monte Carlo simulations were carried out, however the results had higher estimates of the deposited concentrations. Park et al. [12] developed a cold 
neutron depth profiling system at HANARO. The energy distribution was calculated using Monte Carlo simulation code MCStas, and the results were well-matched with the reference values with $1 \%$ and $3.4 \%$ errors. Run-dong Li et al. [13] developed a neutron depth profiling facility at China Mianyang Research Reactor (CMRR). The cold neutron depth profiling measurement of $\mathrm{B}^{10}$ showed consistency with the Standard Reference Materials SRM-2137 values giving rise to the validity of the developed NDP facility.

Y. Sunitha et al. [14] investigated the lithium ion battery by depth profiling the lithium present in electrode materials. The two used methods targeted the nuclear reactions $\mathrm{Li}^{7}(\mathrm{p}, \gamma) \mathrm{Be}^{8}$, and $\mathrm{Li}^{7}(\mathrm{p}, \alpha) \mathrm{He}^{4}$, respectively. Other impurities interact with protons giving rise to interference in both methods. The second method witnessed the least interference at angle $90^{\circ}$, however both methods proved their eligibility. L. Werner et al. [3] tested the depth profiling capabilities of N4DP by depth profiling borofloat glass. The depth resolution for graphite reference sample was simulated using GEANT4. NDP identified different properties on different surfaces.

\section{MODEL DESCRIPTION}

In order to train an ANN, data were generated using MCNP6. The model consisted of a thermal disc neutron source of radius $3 \mathrm{~cm}$ directed towards a boro-silicate sample of $3.5 \mu \mathrm{m}$ thickness. The distance between the neutron source and the sample was $1 \mathrm{~cm}$. A silicon detector was placed at distance of $2 \mathrm{~cm}$ from the sample. The silicon detector was assumed to be of $3 \mathrm{~cm}$ radius and $1 \mathrm{~cm}$ thickness. The sample was divided into ten layers varying in random boron concentrations from 0 to 1. A detailed schematic of the MCNP6 model used in the data generation is illustrated in figure 1.

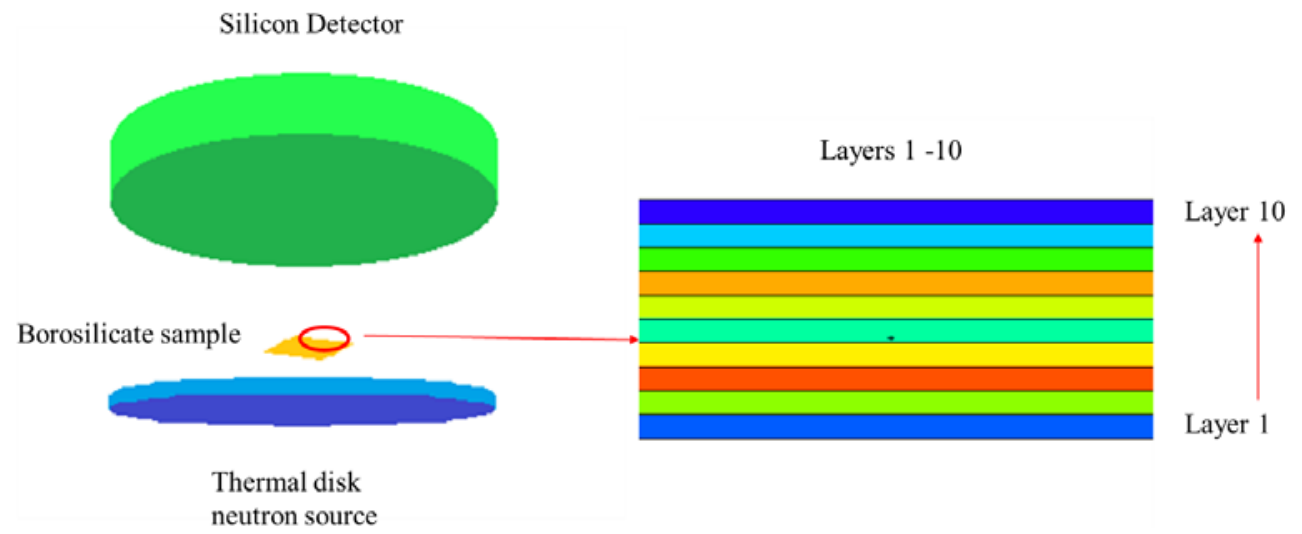

Figure 1. A scheme for the MCNP6 model used for data generation

Readings from the detector are taken as the input features for the developed ANN models while the boron concentrations were taken as the target outputs from the ANN models. The average standard deviation associated with the MCNP6 output varied from 0.01 to 0.04 . Since each sample was divided into ten layers, it was possible to develop ten ANN models (one for each layer) in order to obtain higher regression accuracy in which each model has the same input features; however, only one output corresponding to the layer required to be obtained from the sample as shown in figure 2. Due to the huge run time needed for such computations for data generated, only 150 samples were used in training and testing the developed models in which $5 \%$ of the data set was used as test set for the developed model. 


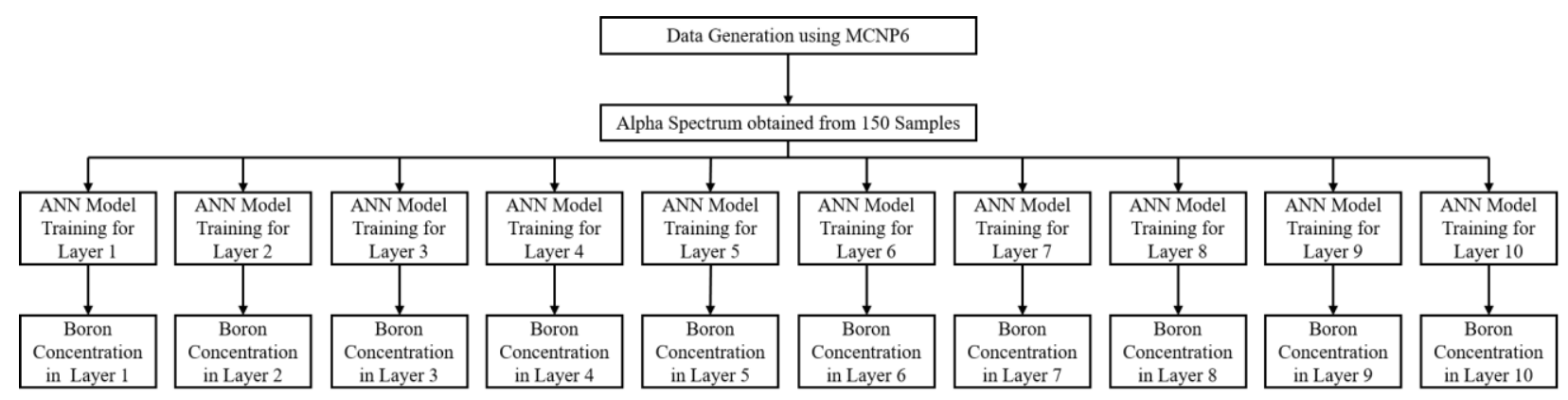

Figure 2. Flow chart describing the employed methodology

\section{RESULTS AND DISCUSSION}

As mentioned earlier in the methodology section, ten models were developed, each of them representing a layer in the investigated sample, hence regression metrics were reported for the ten models as listed in table I. It was shown from table I that the total regression score of the ten models varies from 0.94 to 0.99 , which illustrates the capability of each model to predict the boron concentration from the corresponding alpha spectra for each layer. Also, it was found that the test regression scores are not far behind the training regression scores indicating the models capability of generalizing over unknown samples.

Table I. Training, Validation, Test, and Total R scores for each of the 10 layers and their corresponding Performance

\begin{tabular}{|l|l|l|l|l|l|}
\hline $\begin{array}{l}\text { Layer } \\
\text { Number }\end{array}$ & $\begin{array}{l}\text { Training } \\
\text { R Score }\end{array}$ & $\begin{array}{l}\text { Validation } \\
\text { R Score }\end{array}$ & $\begin{array}{l}\text { Test R } \\
\text { Score }\end{array}$ & $\begin{array}{l}\text { Total R } \\
\text { Score }\end{array}$ & $\begin{array}{l}\text { Performance } \\
\text { (MSE) }\end{array}$ \\
\hline Layer 1 & 0.9676 & 0.80756 & 0.8321 & 0.95452 & $4.2422 \mathrm{E}-08$ \\
\hline Layer 2 & 0.96401 & 0.87366 & 0.85039 & 0.95055 & $1.2050 \mathrm{E}-07$ \\
\hline Layer 3 & 0.99446 & 0.9822 & 0.87706 & 0.9863 & $1.9907 \mathrm{E}-08$ \\
\hline Layer 4 & 0.94798 & 0.85075 & 0.85249 & 0.94062 & $5.6781 \mathrm{E}-08$ \\
\hline Layer 5 & 0.97018 & 0.92336 & 0.91926 & 0.9645 & $6.0627 \mathrm{E}-08$ \\
\hline Layer 6 & 0.97504 & 0.9358 & 0.92554 & 0.96992 & $3.4637 \mathrm{E}-08$ \\
\hline Layer 7 & 0.97509 & 0.95432 & 0.92856 & 0.96847 & $2.4682 \mathrm{E}-08$ \\
\hline Layer 8 & 0.98922 & 0.91932 & 0.93593 & 0.98013 & $5.5935 \mathrm{E}-08$ \\
\hline Layer 9 & 0.99113 & 0.90188 & 0.91046 & 0.98118 & $6.9505 \mathrm{E}-08$ \\
\hline Layer 10 & 0.99759 & 0.95401 & 0.96137 & 0.99157 & $6.6177 \mathrm{E}-08$ \\
\hline
\end{tabular}

The developed models capability was tested over a boro-silicate sample of $3.5 \mu \mathrm{m}$ thickness and 32 $\mathrm{mm}$ diameter. The sample is widely used in NDP tests. Originally, the sample consists of 61 layers varying in boron concentration as shown in figure 3 . The developed models were trained over ten layer samples; hence the reference sample was first condensed into ten layer sample instead of 61 layers. Then, the outcome alpha spectrum was obtained from MCNP6 computations. After that the alpha spectrum was fed to the developed models to predict the values of boron concentration for each of the ten layers as shown in figure 4. A difference has been noticed between the ANN model prediction and the true value in the $7^{\text {th }}$ layer of the investigated sample. This difference is due to the fact that ANN trains over the mean squared error (MSE) loss function. Hence, some data points might have larger 
error than others. However, the low MSE shown in table I indicates that such point in figure 4 can be considered as an abnormality due to the low size of the training data set.

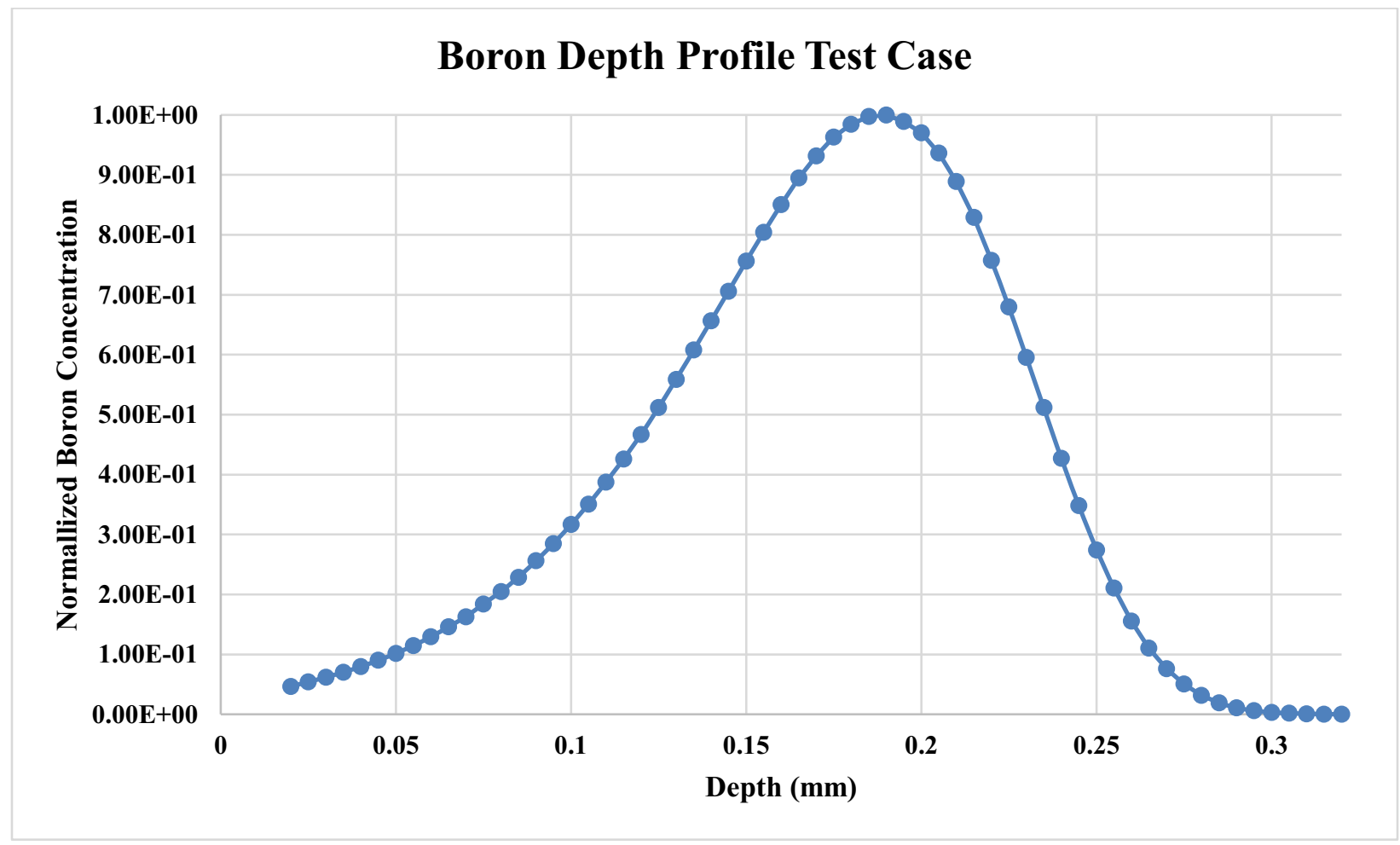

Figure 3. Boron Depth Profile Test Case

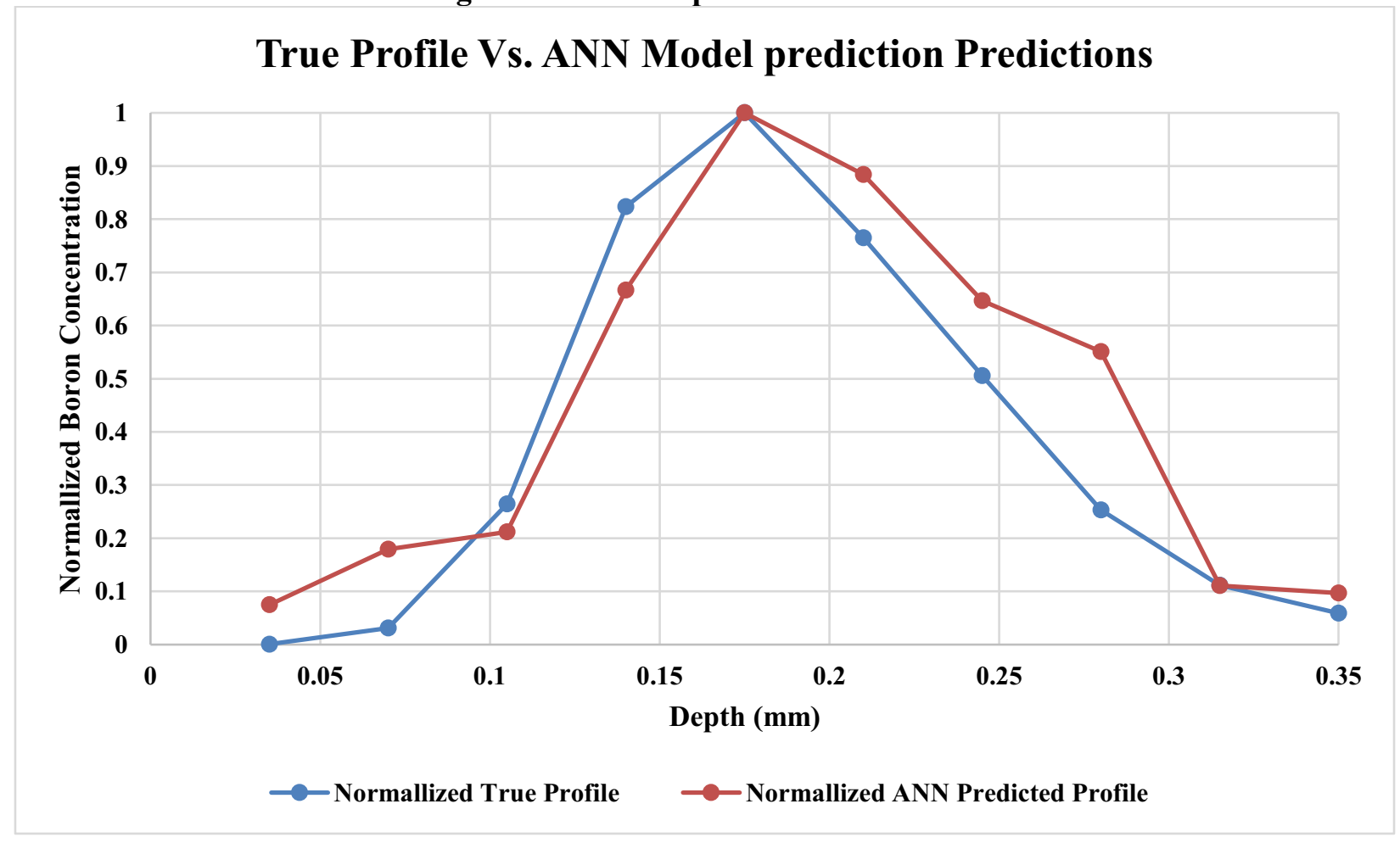


Figure 4. A comparison between the test case original results and the developed ANN results

\section{CONCLUSIONS}

In this work, ten ANN models were developed to predict the boron-depth profile for different borosilicate samples. Each of the developed models was responsible for predicting the boron concentration for a certain layer. The models showed their capability of predicting the boron concentration from different alpha spectra. Limited resources and limited number of samples were used in this work. However, it proved that ANNs are capable of solving neutron depth profiling quantification problem. Future work should include larger training, validation and test data sets in addition to the inclusion of experimentally obtained data in the training process for calibration purposes. Also, the capability of using non-monoenergetic neutron sources such as the neutron spectrum produced in a light water reactor should be investigated for this experiment.

\section{REFERENCES}

[1] J. F. Ziegler, G. W. Cole, J. E. E. Baglin and B.J. Masters, "Boron atom distributions in ionimplanted silicon by the (n, $\left.{ }^{4} \mathrm{He}\right)$ nuclear reaction," Applied Physics Letters, vol. 21(1), pp. 16-17, 1972.

[2] D. Fink,J.P.Biersack and H.Liebl, "Background in (n,p), $(\mathrm{n}, \alpha)$ spectrometry," Ion Implantation: Equipment and Techniques, pp. 318, Germany, Springer Series in Electrophysics, vol. 11, Springer, Berlin, Heidelberg, 1982.

[3] L. Werner, M. Trunk, R. Gernhäuser, R. Gilles, B. Märkisch, Zs. Révay, "The new neutron depth profiling instrument N4DP at the Heinz Maier-Leibnitz Zentrum," Nuclear Inst. and Methods in Physics Research A, vol. 911, pp. 30-36, 2018.

[4] G. P. Lamaze, H. Chen-Mayer, J. K. Langland and R. G. Downing, "Neutron Depth Profiling with the New NIST Cold Neutron Source," Surface and interaction analysis, vol. 25, pp. 217-220, 1997.

[5] J. K. Shultis, "Notes on Neutron Depth Profiling," Manhattan, Kansas, USA, 2003.

[6] M. Kubat, "An Introduction to Machine Learning," University of Miami, Coral Gables, FL, USA: Springer, 2017.

[7] Ivan Nunes da Silva, Danilo Hernane Spatti, Rogerio Andrade Flauzino, Luisa Helena Bartocci Liboni, Silas Franco dos Reis Alves, "Artificial Neural Networks, A Practical Course," University of São Paulo, São Carlos, São Paulo, Brazil: Springer, 2017.

[8] V. Havráhek, V. Hnatowicz, J. Kvítek, J. Vacík and J. Hoffmann, "Neutron depth profiling by large angle coincidence spectrometry," Nuclear Instruments and Methods in Physics Research B, vol. 73, pp. 523-530, 1993.

[9] Kenan Ünlü, Mehmet Saglam, Bernard W. Wehring, "Helium-3 and boron-10 concentration and depth measurements in alloys and semiconductors using NDP," Nuclear Instruments and Methods in Physics Research A, vol. 422, pp. 885-890, 1999.

[10] Sacit M. Çetiner, Kenan Ünlü, "Depth profiling of boron in ultra-shallow junction devices using time-of-flight neutron depth profiling (TOF-NDP)," Nuclear Instruments and Methods in Physics Research A, vol. 579, pp. 148-152, 2007.

[11] R. Bencardino, G Giorginis, D. Sapundjiev, "Preparation of ${ }^{6} \mathrm{LiF}$ deposits and characterization via Monte Carlo Simulations and Neutron Depth Profiling," Nuclear Instruments and Methods in Physics Research A, vol. 709, pp. 72-75, 2012.

[12] B.G. Park, G.M.Sun, H.D.Choi, "Development of cold neutron depth profiling system at HANARO," Nuclear Instruments and Methods in Physics Research A, vol. 752, pp. 20-26, 2014. 
[13] Run-dong Li, Xin Yang, Guan-bo Wang, Hai-feng Dou, Da-zhi Qian, Shu-yu Wang, "Development of neutron depth profiling at CMRR," Nuclear Instruments and Methods in Physics Research A, vol. 788, pp. 1-4, 2015.

[14] Y. Sunitha, Sanjiv Kumar, "Depth profiling Li in electrode materials of lithium ion battery by $\mathrm{Li}^{7}$ $(\mathrm{p}, \gamma) \mathrm{Be}^{8}$ and $\mathrm{Li}^{7}(\mathrm{p}, \alpha) \mathrm{He}^{4}$ nuclear reactions," Nuclear Instruments and Methods in Physics Research B, vol. 400, pp. 22-30, 2017. 